\title{
Microbiology Specimen Domain
}

National Cancer Institute

\section{Source}

National Cancer Institute. Microbiology Specimen Domain. NCI Thesaurus. Code C49602.

A subject domain utilized for the submission of information encompassing and representing data, vocabulary or records related to microbiology specimen. 